\title{
Measurement of the Top Quark Mass with the Collider Detector at Fermilab
}

F. Abe, ${ }^{17}$ H. Akimoto, ${ }^{39}$ A. Akopian, ${ }^{31}$ M. G. Albrow, ${ }^{7}$ A. Amadon, ${ }^{5}$ S. R. Amendolia, ${ }^{27}$ D. Amidei, ${ }^{20}$ J. Antos, ${ }^{33}$ S. Aota,${ }^{37}$ G. Apollinari, ${ }^{31}$ T. Arisawa,${ }^{39}$ T. Asakawa, ${ }^{37}$ W. Ashmanskas,,${ }^{18}$ M. Atac,${ }^{7}$ P. Azzi-Bacchetta,${ }^{25}$ N. Bacchetta, ${ }^{25}$ S. Bagdasarov, ${ }^{31}$ M. W. Bailey, ${ }^{22}$ P. de Barbaro, ${ }^{30}$ A. Barbaro-Galtieri, ${ }^{18}$ V. E. Barnes,${ }^{29}$ B. A. Barnett, ${ }^{15}$ M. Barone, ${ }^{9}$ G. Bauer, ${ }^{19}$ T. Baumann, ${ }^{11}$ F. Bedeschi, ${ }^{27}$ S. Behrends, ${ }^{3}$ S. Belforte, ${ }^{27}$ G. Bellettini,${ }^{27}$ J. Bellinger ${ }^{40}$ D. Benjamin, ${ }^{35}$ J. Bensinger, ${ }^{3}$ A. Beretvas, ${ }^{7}$ J. P. Berge, ${ }^{7}$ J. Berryhill, ${ }^{5}$ S. Bertolucci, ${ }^{9}$ S. Bettelli, ${ }^{27}$ B. Bevensee, ${ }^{26}$ A. Bhatti, ${ }^{31}$ K. Biery, ${ }^{7}$ C. Bigongiari, ${ }^{27}$ M. Binkley, ${ }^{7}$ D. Bisello, ${ }^{25}$ R. E. Blair, ${ }^{1}$ C. Blocker,${ }^{3}$ K. Bloom,${ }^{20}$ S. Blusk,${ }^{30}$ A. Bodek,${ }^{30}$ W. Bokhari, ${ }^{26}$ G. Bolla, ${ }^{29}$ Y. Bonushkin,,${ }^{4}$ D. Bortoletto, ${ }^{29}$ J. Boudreau,${ }^{28}$ L. Breccia, ${ }^{2}$ C. Bromberg, ${ }^{21}$ N. Bruner, ${ }^{22}$ R. Brunetti, ${ }^{2}$ E. Buckley-Geer, ${ }^{7}$ H. S. Budd, ${ }^{30}$ K. Burkett, ${ }^{11}$ G. Busetto, ${ }^{25}$ A. Byon-Wagner, ${ }^{7}$ K. L. Byrum, ${ }^{1}$ M. Campbell, ${ }^{20}$ A. Caner,${ }^{27}$ W. Carithers, ${ }^{18}$ D. Carlsmith, ${ }^{40}$ J. Cassada,${ }^{30}$ A. Castro, ${ }^{25}$ D. Cauz,${ }^{36}$ A. Cerri, ${ }^{27}$ P. S. Chang, ${ }^{33}$ P. T. Chang, ${ }^{33}$ H. Y. Chao,${ }^{33}$ J. Chapman, ${ }^{20}$ M.-T. Cheng, ${ }^{33}$ M. Chertok, ${ }^{34}$ G. Chiarelli, ${ }^{27}$ C. N. Chiou, ${ }^{33}$ F. Chlebana, ${ }^{7}$ L. Christofek, ${ }^{13}$ R. Cropp,${ }^{14}$ M. L. Chu, ${ }^{33}$ S. Cihangir, ${ }^{7}$ A. G. Clark, ${ }^{10}$ M. Cobal, ${ }^{27}$ E. Cocca,${ }^{27}$ M. Contreras,,${ }^{5}$ J. Conway, ${ }^{32}$ J. Cooper, ${ }^{7}$ M. Cordelli, ${ }^{9}$ D. Costanzo, ${ }^{27}$ C. Couyoumtzelis, ${ }^{10}$ D. Cronin-Hennessy, ${ }^{6}$ R. Culbertson, ${ }^{5}$ D. Dagenhart,${ }^{38}$ T. Daniels, ${ }^{19}$ F. DeJongh, ${ }^{7}$

S. Dell'Agnello, ${ }^{9}$ M. Dell'Orso, ${ }^{27}$ R. Demina,${ }^{7}$ L. Demortier,${ }^{31}$ M. Deninno, ${ }^{2}$ P. F. Derwent, ${ }^{7}$ T. Devlin, ${ }^{32}$ J. R. Dittmann, ${ }^{6}$ S. Donati, ${ }^{27}$ J. Done, ${ }^{34}$ T. Dorigo, ${ }^{25}$ N. Eddy, ${ }^{13}$ K. Einsweiler, ${ }^{18}$ J. E. Elias, ${ }^{7}$ R. Ely, ${ }^{18}$ E. Engels, Jr., ${ }^{28}$ W. Erdmann, ${ }^{7}$ D. Errede, ${ }^{13}$ S. Errede, ${ }^{13}$ Q. Fan, ${ }^{30}$ R. G. Feild, ${ }^{41}$ Z. Feng, ${ }^{15}$ C. Ferretti, ${ }^{27}$ I. Fiori, ${ }^{2}$ B. Flaugher, ${ }^{7}$ G.W. Foster, ${ }^{7}$ M. Franklin, ${ }^{11}$ J. Freeman, ${ }^{7}$ J. Friedman, ${ }^{19}$ H. Frisch, ${ }^{5}$ Y. Fukui, ${ }^{17}$ S. Gadomski, ${ }^{14}$ S. Galeotti, ${ }^{27}$ M. Gallinaro, ${ }^{26}$ O. Ganel, ${ }^{35}$ M. Garcia-Sciveres, ${ }^{18}$ A. F. Garfinkel, ${ }^{29}$ C. Gay, ${ }^{41}$ S. Geer, ${ }^{7}$ D. W. Gerdes,${ }^{20}$ P. Giannetti ${ }^{27}$ N. Giokaris, ${ }^{31}$ P. Giromini, ${ }^{9}$ G. Giusti, ${ }^{27}$ M. Gold,${ }^{22}$ A. Gordon, ${ }^{11}$ A. T. Goshaw,${ }^{6}$ Y. Gotra ${ }^{28}$ K. Goulianos,${ }^{31}$ H. Grassmann, ${ }^{36}$ L. Groer, ${ }^{32}$ C. Grosso-Pilcher, ${ }^{5}$ G. Guillian, ${ }^{20}$ J. Guimaraes da Costa, ${ }^{15}$ R. S. Guo, ${ }^{33}$ C. Haber, ${ }^{18}$

E. Hafen, ${ }^{19}$ S. R. Hahn, ${ }^{7}$ R. Hamilton, ${ }^{11}$ T. Handa,${ }^{12}$ R. Handler, ${ }^{40}$ W. Hao, ${ }^{35}$ F. Happacher, ${ }^{9}$ K. Hara,${ }^{37}$

A. D. Hardman, ${ }^{29}$ R. M. Harris, ${ }^{7}$ F. Hartmann, ${ }^{16}$ J. Hauser ${ }^{4}$ E. Hayashi, ${ }^{37}$ J. Heinrich ${ }^{26}$ A. Heiss,${ }^{16}$ B. Hinrichsen, ${ }^{14}$

K. D. Hoffman, ${ }^{29}$ M. Hohlmann, ${ }^{5}$ C. Holck, ${ }^{26}$ R. Hollebeek, ${ }^{26}$ L. Holloway, ${ }^{13}$ Z. Huang, ${ }^{20}$ B. T. Huffman, ${ }^{28}$ R. Hughes, ${ }^{23} \mathrm{~J}$. Huston, ${ }^{21} \mathrm{~J}$. Huth, ${ }^{11} \mathrm{H}$. Ikeda,${ }^{37} \mathrm{M}$. Incagli, ${ }^{27} \mathrm{~J}$. Incandela,${ }^{7} \mathrm{G}$. Introzzi, ${ }^{27} \mathrm{~J}$. Iwai, ${ }^{39} \mathrm{Y}$. Iwata, ${ }^{12}$ E. James,${ }^{20}$ H. Jensen, ${ }^{7}$ U. Joshi, ${ }^{7}$ E. Kajfasz, ${ }^{25}$ H. Kambara, ${ }^{10}$ T. Kamon, ${ }^{34}$ T. Kaneko, ${ }^{37}$ K. Karr,${ }^{38}$ H. Kasha,${ }^{41}$ Y. Kato, ${ }^{24}$ T. A. Keaffaber, ${ }^{29}$ K. Kelley, ${ }^{19}$ R. D. Kennedy, ${ }^{7}$ R. Kephart, ${ }^{7}$ D. Kestenbaum, ${ }^{11}$ D. Khazins, ${ }^{6}$ T. Kikuchi, ${ }^{37}$ B. J. Kim, ${ }^{27}$ H. S. Kim, ${ }^{14}$ S. H. Kim, ${ }^{37}$ Y. K. Kim,${ }^{18}$ L. Kirsch, ${ }^{3}$ S. Klimenko,${ }^{8}$ D. Knoblauch, ${ }^{16}$ P. Koehn, ${ }^{23}$

A. Köngeter, ${ }^{16}$ K. Kondo, ${ }^{37}$ J. Konigsberg, ${ }^{8}$ K. Kordas, ${ }^{14}$ A. Korytov, ${ }^{8}$ E. Kovacs, ${ }^{1}$ W. Kowald, ${ }^{6}$ J. Kroll, ${ }^{26}$ M. Kruse, ${ }^{30}$ S. E. Kuhlmann, ${ }^{1}$ E. Kuns, ${ }^{32}$ K. Kurino, ${ }^{12}$ K. Kuwabara, ${ }^{37}$ A. T. Laasanen, ${ }^{29}$ S. Lami, ${ }^{27}$ S. Lammel, ${ }^{7}$ J. I. Lamoureux, ${ }^{3}$ M. Lancaster, ${ }^{18}$ M. Lanzoni, ${ }^{27}$ G. Latino, ${ }^{27}$ T. LeCompte, ${ }^{1}$ S. Leone,${ }^{27}$ J. D. Lewis, ${ }^{7}$ M. Lindgren, ${ }^{4}$ T. M. Liss, ${ }^{13}$ J. B. Liu, ${ }^{30}$ Y. C. Liu ${ }^{33}$ N. Lockyer, ${ }^{26}$ O. Long, ${ }^{26}$ M. Loreti, ${ }^{25}$ D. Lucchesi, ${ }^{27}$ P. Lukens, ${ }^{7}$ S. Lusin, ${ }^{40}$ J. Lys, ${ }^{18}$ K. Maeshima, ${ }^{7}$ P. Maksimovic, ${ }^{11}$ M. Mangano, ${ }^{27}$ M. Mariotti, ${ }^{25}$ J. P. Marriner, ${ }^{7}$ G. Martignon, ${ }^{25}$ A. Martin, ${ }^{41}$ J. A. J. Matthews, ${ }^{22}$ P. Mazzanti, ${ }^{2}$ K. McFarland,${ }^{30}$ P. McIntyre, ${ }^{34}$ P. Melese,${ }^{31}$ M. Menguzzato, ${ }^{25}$ A. Menzione, ${ }^{27}$ E. Meschi, ${ }^{27}$ S. Metzler ${ }^{26}$ C. Miao, ${ }^{20}$ T. Miao,${ }^{7}$ G. Michail,${ }^{11}$ R. Miller, ${ }^{21}$ H. Minato ${ }^{37}$ S. Miscetti, ${ }^{9}$ M. Mishina, ${ }^{17}$ S. Miyashita, ${ }^{37}$ N. Moggi, ${ }^{27}$ E. Moore, ${ }^{22}$ Y. Morita, ${ }^{17}$ A. Mukherjee, ${ }^{7}$ T. Muller, ${ }^{16}$ P. Murat,${ }^{27}$ S. Murgia, ${ }^{21}$ M. Musy,${ }^{36}$

H. Nakada, ${ }^{37}$ T. Nakaya,${ }^{5}$ I. Nakano, ${ }^{12}$ C. Nelson, ${ }^{7}$ D. Neuberger, ${ }^{16}$ C. Newman-Holmes, ${ }^{7}$ C.-Y.P. Ngan, ${ }^{19}$

L. Nodulman, ${ }^{1}$ A. Nomerotski, ${ }^{8}$ S. H. Oh, ${ }^{6}$ T. Ohmoto, ${ }^{12}$ T. Ohsugi, ${ }^{12}$ R. Oishi, ${ }^{37}$ M. Okabe, ${ }^{37}$ T. Okusawa, ${ }^{24}$ J. Olsen, ${ }^{40}$ C. Pagliarone,${ }^{27}$ R. Paoletti, ${ }^{27}$ V. Papadimitriou, ${ }^{35}$ S. P. Pappas,${ }^{41}$ N. Parashar, ${ }^{27}$ A. Parri, ${ }^{9}$ J. Patrick,${ }^{7}$ G. Pauletta, ${ }^{36}$ M. Paulini, ${ }^{18}$ A. Perazzo, ${ }^{27}$ L. Pescara,${ }^{25}$ M. D. Peters, ${ }^{18}$ T. J. Phillips,${ }^{6}$ G. Piacentino,${ }^{27}$ M. Pillai, ${ }^{30}$ K. T. Pitts, ${ }^{7}$ R. Plunkett, ${ }^{7}$ A. Pompos,${ }^{29}$ L. Pondrom,${ }^{40}$ J. Proudfoot, ${ }^{1}$ F. Ptohos,${ }^{11}$ G. Punzi, ${ }^{27}$ K. Ragan, ${ }^{14}$ D. Reher, ${ }^{18}$ M. Reischl, ${ }^{16}$ A. Ribon, ${ }^{25}$ F. Rimondi, ${ }^{2}$ L. Ristori, ${ }^{27}$ W. J. Robertson, ${ }^{6}$ A. Robinson, ${ }^{14}$ T. Rodrigo, ${ }^{27}$ S. Rolli, ${ }^{38}$ L. Rosenson, ${ }^{19}$ R. Roser, ${ }^{13}$ T. Saab, ${ }^{14}$ W. K. Sakumoto, ${ }^{30}$ D. Saltzberg, ${ }^{4}$ A. Sansoni, ${ }^{9}$ L. Santi, ${ }^{36}$ H. Sato,${ }^{37}$ P. Schlabach, ${ }^{7}$ E. E. Schmidt, ${ }^{7}$ M. P. Schmidt, ${ }^{41}$ A. Scott ${ }^{4}$ A. Scribano, ${ }^{27}$ S. Segler ${ }^{7}$ S. Seidel, ${ }^{22}$ Y. Seiya, ${ }^{37}$ F. Semeria, ${ }^{2}$ T. Shah, ${ }^{19}$ M. D. Shapiro, ${ }^{18}$ N. M. Shaw,${ }^{29}$ P. F. Shepard, ${ }^{28}$ T. Shibayama,${ }^{37}$ M. Shimojima,${ }^{37}$ M. Shochet, ${ }^{5}$ J. Siegrist, ${ }^{18}$ A. Sill, ${ }^{35}$ P. Sinervo, ${ }^{14}$ P. Singh, ${ }^{13}$ K. Sliwa ${ }^{38}$ C. Smith, ${ }^{15}$ F. D. Snider, ${ }^{15}$ J. Spalding, ${ }^{7}$

T. Speer, ${ }^{10}$ P. Sphicas, ${ }^{19}$ F. Spinella, ${ }^{27}$ M. Spiropulu,${ }^{11}$ L. Spiegel, ${ }^{7}$ L. Stanco,${ }^{25}$ J. Steele, ${ }^{40}$ A. Stefanini,${ }^{27}$

R. Ströhmer, ${ }^{7, *}$ J. Strologas, ${ }^{13}$ F. Strumia,${ }^{10}$ D. Stuart, ${ }^{7}$ K. Sumorok,,${ }^{19}$ J. Suzuki, ${ }^{37}$ T. Suzuki, ${ }^{37}$ T. Takahashi, ${ }^{24}$

T. Takano, ${ }^{24} \mathrm{R}$. Takashima, ${ }^{12} \mathrm{~K}$. Takikawa, ${ }^{37} \mathrm{M}$. Tanaka, ${ }^{37}$ B. Tannenbaum, ${ }^{4}$ F. Tartarelli, ${ }^{27} \mathrm{~W}$. Taylor, ${ }^{14}$ M. Tecchio, ${ }^{20}$ P. K. Teng, ${ }^{33}$ Y. Teramoto,${ }^{24}$ K. Terashi, ${ }^{37}$ S. Tether, ${ }^{19}$ D. Theriot,,${ }^{7}$ T. L. Thomas, ${ }^{22}$ R. Thurman-Keup, ${ }^{1}$ 
M. Timko, ${ }^{38}$ P. Tipton,${ }^{30}$ A. Titov,${ }^{31}$ S. Tkaczyk,${ }^{7}$ D. Toback,${ }^{5}$ K. Tollefson, ${ }^{30}$ A. Tollestrup,${ }^{7}$ H. Toyoda,${ }^{24}$ W. Trischuk, ${ }^{14}$ J.F. de Troconiz,${ }^{11}$ S. Truitt, ${ }^{20}$ J. Tseng, ${ }^{19}$ N. Turini, ${ }^{27}$ T. Uchida, ${ }^{37}$ F. Ukegawa, ${ }^{26}$ J. Valls,${ }^{32}$ S. C. van den Brink, ${ }^{15}$ S. Vejcik III,${ }^{20}$ G. Velev, ${ }^{27}$ I. Volobouev, ${ }^{18}$ R. Vidal,${ }^{7}$ R. Vilar, ${ }^{7, *}$ D. Vucinic, ${ }^{19}$ R. G. Wagner, ${ }^{1}$ R.L. Wagner, ${ }^{7}$ J. Wahl,${ }^{5}$ N. B. Wallace, ${ }^{27}$ A. M. Walsh, ${ }^{32}$ C. Wang,,${ }^{6}$ C. H. Wang, ${ }^{33}$ M. J. Wang, ${ }^{33}$ A. Warburton, ${ }^{14}$ T. Watanabe, ${ }^{37}$ T. Watts,${ }^{32}$ R. Webb,${ }^{34}$ C. Wei, ${ }^{6}$ H. Wenzel, ${ }^{16}$ W. C. Wester III, ${ }^{7}$ A. B. Wicklund, ${ }^{1}$ E. Wicklund, ${ }^{7}$ R. Wilkinson, ${ }^{26}$ H. H. Williams, ${ }^{26}$ P. Wilson, ${ }^{7}$ B. L. Winer, ${ }^{23}$ D. Winn, ${ }^{20}$ D. Wolinski, ${ }^{20}$ J. Wolinski, ${ }^{21}$ S. Worm, ${ }^{22}$ X. Wu, ${ }^{10}$ J. Wyss,${ }^{27}$ A. Yagil, ${ }^{7}$ W. Yao ${ }^{18}$ K. Yasuoka, ${ }^{37}$ G. P. Yeh, ${ }^{7}$ P. Yeh, ${ }^{33}$ J. Yoh, ${ }^{7}$ C. Yosef, ${ }^{21}$ T. Yoshida, ${ }^{24}$ I. Yu, ${ }^{7}$ A. Zanetti, ${ }^{36}$ F. Zetti, ${ }^{27}$ and S. Zucchelli ${ }^{2}$

(CDF Collaboration)

${ }^{1}$ Argonne National Laboratory, Argonne, Illinois 60439

${ }^{2}$ Istituto Nazionale di Fisica Nucleare, University of Bologna, I-40127 Bologna, Italy

${ }^{3}$ Brandeis University, Waltham, Massachusetts 02254

${ }^{4}$ University of California at Los Angeles, Los Angeles, California 90024

${ }^{5}$ University of Chicago, Chicago, Illinois 60637

${ }^{6}$ Duke University, Durham, North Carolina 27708

${ }^{7}$ Fermi National Accelerator Laboratory, Batavia, Illinois 60510

${ }^{8}$ University of Florida, Gainesville, Florida 32611

${ }^{9}$ Laboratori Nazionali di Frascati, Istituto Nazionale di Fisica Nucleare, I-00044 Frascati, Italy

${ }^{10}$ University of Geneva, CH-1211 Geneva 4, Switzerland

${ }^{11}$ Harvard University, Cambridge, Massachusetts 02138

${ }^{12}$ Hiroshima University, Higashi-Hiroshima 724, Japan

${ }^{13}$ University of Illinois, Urbana, Illinois 61801

${ }^{14}$ Institute of Particle Physics, McGill University, Montreal H3A 2T8, Canada and University of Toronto, Toronto M5S 1A7, Canada

${ }^{15}$ The Johns Hopkins University, Baltimore, Maryland 21218

${ }^{16}$ Institut für Experimentelle Kernphysik, Universität Karlsruhe, 76128 Karlsruhe, Germany

${ }^{17}$ National Laboratory for High Energy Physics (KEK), Tsukuba, Ibaraki 305, Japan

${ }^{18}$ Ernest Orlando Lawrence Berkeley National Laboratory, Berkeley, California 94720

${ }^{19}$ Massachusetts Institute of Technology, Cambridge, Massachusetts 02139

${ }^{20}$ University of Michigan, Ann Arbor, Michigan 48109

${ }^{21}$ Michigan State University, East Lansing, Michigan 48824

${ }^{22}$ University of New Mexico, Albuquerque, New Mexico 87131

${ }^{23}$ The Ohio State University, Columbus, Ohio 43210

${ }^{24}$ Osaka City University, Osaka 588, Japan

${ }^{25}$ Universita di Padova, Istituto Nazionale di Fisica Nucleare, Sezione di Padova, I-35131 Padova, Italy

${ }^{26}$ University of Pennsylvania, Philadelphia, Pennsylvania 19104

${ }^{27}$ Istituto Nazionale di Fisica Nucleare, University and Scuola Normale Superiore of Pisa, I-56100 Pisa, Italy

${ }^{28}$ University of Pittsburgh, Pittsburgh, Pennsylvania 15260

${ }^{29}$ Purdue University, West Lafayette, Indiana 47907

${ }^{30}$ University of Rochester, Rochester, New York 14627

${ }^{31}$ Rockefeller University, New York, New York 10021

${ }^{32}$ Rutgers University, Piscataway, New Jersey 08855

${ }^{33}$ Academia Sinica, Taipei, Taiwan 11530, Republic of China

${ }^{34}$ Texas A\&M University, College Station, Texas 77843

${ }^{35}$ Texas Tech University, Lubbock, Texas 79409

${ }^{36}$ Istituto Nazionale di Fisica Nucleare, University of Trieste/Udine, Italy

${ }^{37}$ University of Tsukuba, Tsukuba, Ibaraki 315, Japan

${ }^{38}$ Tufts University, Medford, Massachusetts 02155

${ }^{39}$ Waseda University, Tokyo 169, Japan

${ }^{40}$ University of Wisconsin, Madison, Wisconsin 53706

${ }^{41}$ Yale University, New Haven, Connecticut 06520

(Received 12 October 1998)

We present a new measurement of the top quark mass in $t \bar{t}$ events in which both $W$ bosons from top quarks decay into leptons $(e \nu, \mu \nu)$. We use events collected by the CDF experiment from $p \bar{p}$ collisions at $\sqrt{s}=1.8 \mathrm{TeV}$ at the Tevatron collider. We measure a top quark mass of $167.4 \pm 10.3$ (stat) \pm 4.8 (syst) $\mathrm{GeV} / c^{2}$ from a sample of eight events. We combine this result with previous $\mathrm{CDF}$ measurements in other decay channels to obtain a final mass value of $176.0 \pm$ $6.5 \mathrm{GeV} / c^{2}$. [S0031-9007(98)08195-2]

PACS numbers: 14.65.Ha, 13.85.Ni, 13.85.Qk 
The top quark, the sixth and final quark of the standard model, is found to have a surprisingly large mass. A precise measurement of this mass is important in testing the consistency of the standard model with experimental data. In addition, precise $W$ and top mass measurements can provide information on the mass of the Higgs boson, which is a remnant of the mechanism that gives rise to spontaneous electroweak symmetry breaking. Using $109 \mathrm{pb}^{-1}$ of data accumulated by the CDF experiment at the Fermilab Tevatron from 1992 through 1995, we report an improved measurement of the top quark mass using dilepton events originating predominantly from $t \bar{t} \rightarrow W^{+} b W^{-} \bar{b} \rightarrow\left(\ell^{+} \nu b\right)\left(\ell^{-} \bar{\nu} \bar{b}\right)$, where $\ell=e$ or $\mu$. This measurement supersedes our previously reported result in the dilepton channel [1]. The previous result was obtained by comparing data with Monte Carlo simulation of $t \bar{t}$ events for two kinematic variables, the $b$-jet energies and the invariant masses of the lepton and $b$-jet systems. Here we make use of all available information in the event and obtain a more precise measurement. We combine the result from the dilepton channel with those from the lepton plus jets channel $\left[t \bar{t} \rightarrow W^{+} b W^{-} \bar{b} \rightarrow\right.$ $\left.\left(\ell^{+} \nu b\right)\left(q \bar{q}^{\prime} \bar{b}\right)\right][2]$ and the all-hadronic channel $[t \bar{t} \rightarrow$ $\left(q \bar{q}^{\prime} b\right)\left(q \bar{q}^{\prime} \bar{b}\right)$ ] [3] to obtain a final top quark mass value from the CDF Run I data. The only other direct measurements are reported by the D0 experiment at the Tevatron $[4,5]$.

The CDF detector consists of a magnetic spectrometer surrounded by calorimeters and muon chambers [6]. A four-layer silicon vertex detector (SVX) [7], located immediately outside the beam pipe, is surrounded by the central tracking chamber (CTC) which is inside a $1.4 \mathrm{~T}$ superconducting solenoid. This tracking system is used to measure the momenta of charged particles. Electromagnetic and hadronic calorimeters, located outside the CTC, are segmented in projective towers and cover the pseudorapidity region $|\eta|<4.2$ [8]. They are used to identify electron and photon candidates and jets, and are used to measure the missing transverse energy $\left(\mathbb{E}_{T}\right)$ which can indicate the presence of energetic neutrinos. Outside the calorimeters, drift chambers in the region $|\eta|<1.0$ provide for muon identification. A three-level trigger selects events that contain a high $P_{T}$ electron or muon for this analysis.

We apply the same event selection criteria and use identical background calculations as those employed in the previous mass analyses of the dilepton channel [1]. We require two high transverse momentum $\left(P_{T}>20 \mathrm{GeV} / c\right)$ oppositely charged leptons $(e$ or $\mu)$ in the central detector region $(|\eta|<1)$, with at least one of them well isolated from nearby tracks and calorimeter activity. To reject $Z \rightarrow \ell^{+} \ell^{-} X$ events we require that the dilepton invariant mass, $M_{e e}$ or $M_{\mu \mu}$, be outside the interval $75-105 \mathrm{GeV} / c^{2}$, and remove events containing an isolated photon with $E_{T}>10 \mathrm{GeV}$ if they are consistent with radiative $Z$ decays. We require at least two jets in the region $|\eta|<2.0$, each with observed $E_{T}>10 \mathrm{GeV}$ in a cone radius $\Delta R=\sqrt{\Delta \eta^{2}+\Delta \phi^{2}}=0.4$, and require $\left|\not_{T}\right|>25 \mathrm{GeV}$ as a signature for missing neutrinos. To reject events in which $\not_{T}$ is due to lepton or jet energy mismeasurements we require $\left|\mathscr{E}_{T}\right|>50 \mathrm{GeV}$ if $\not_{T}$ is close to a lepton or a jet $\left[\Delta \phi\left(\mathbb{E}_{T}, \ell\right.\right.$ or $\left.\left.j\right)<20^{\circ}\right]$. Finally, we require $H_{T}>170 \mathrm{GeV}$, where $H_{T}$ is the scalar sum of the $\left|P_{T}\right|$ of the two leptons ( $E_{T}$ for electrons), the $E_{T}$ of the two highest $E_{T}$ jets and $\left|\mathscr{E}_{T}\right|$. We obtain a sample of eight candidate events. The expected background of $1.3 \pm 0.3$ events consists of events in which a track or a jet is misidentified as a lepton (0.29 events), Drell-Yan production (0.35 events), $W W$ production ( 0.24 events), $Z \rightarrow \tau \tau$ decays ( 0.26 events), and $Z \rightarrow \mu \mu$ decays in which $\mu$ tracks are mismeasured ( 0.20 events).

For the top mass measurement the jet energies are corrected for losses in cracks between detector components, absolute energy scale, contributions from the underlying event and multiple interactions, and losses outside the clustering cone. These corrections are determined from a combination of Monte Carlo simulation and data [2]. Additional energy corrections are applied to jets containing a muon to take into account the low calorimeter response to muons and missing neutrinos from semileptonic decays of heavy quarks in the jets. We obtain a corrected $\not_{T}$ by correcting each of its components, i.e., the jets, the leptons, and the energy which has not been clustered into jets.

The procedure used here has two steps: we reconstruct each event to obtain a top mass estimate for the event, then we apply a likelihood method to obtain an overall mass value in the presence of background. Each candidate event is reconstructed according to the $t \bar{t}$ decay hypothesis in the dilepton channel:

$$
\begin{aligned}
& t \rightarrow W^{+} b \rightarrow \ell_{1}^{+} \nu_{1} b, \\
& \bar{t} \rightarrow W^{-} \bar{b} \rightarrow \ell_{2}^{-} \bar{\nu}_{2} \bar{b} .
\end{aligned}
$$

The two highest $E_{T}$ jets in the event are assumed to be the $b$ jets from top decays. We assume the $b$-jet mass to be $5 \mathrm{GeV} / c^{2}$. After applying the invariant mass constraints $m\left(\ell_{1} \nu_{1}\right)=m\left(\ell_{2} \bar{\nu}_{2}\right)=m_{W}$ and $m\left(\ell_{1} \nu_{1} b\right)=$ $m\left(\ell_{2} \bar{\nu}_{2} \bar{b}\right)$, the system remains underconstrained due to the two unmeasured neutrinos. Therefore, for any assumed top mass value $m_{t}$, we use a weighting technique to determine a function, $f\left(m_{t}\right)$, from which we extract a top mass value [5,9]. We proceed as follows. We assume a top quark mass $\left(m_{t}\right)$ and the two neutrino $\eta$ values $\left(\eta_{1}\right.$, $\eta_{2}$; see below) and solve for the neutrino momenta, up to a fourfold ambiguity [two $P_{z}(\nu)$ choices for each $\nu$ ] for each of the two jet charged-lepton pairings. We then assign a weight to each solution by comparing $\mathbb{H}_{T}{ }^{p}$, the sum of the neutrino transverse momenta for that solution, to $\mathbb{E}_{T}{ }^{m}$, the measured missing transverse energy after proper correction:

$$
g\left(m_{t}, \eta_{1}, \eta_{2}\right)=\exp \left(-\frac{\left(\not_{T}^{p}-\not \mathbb{E}_{T}^{m}\right)^{2}}{2 \sigma^{2}}\right),
$$

where $\sigma$ is the $\not_{T}$ resolution for that event (see below).

For each choice of $m_{t}, \eta_{1}$, and $\eta_{2}$ we take into account the detector resolution for jets and leptons 
by sampling (i.e., fluctuating) the measured quantities many times according to their resolutions. The Gaussian resolutions for electrons and muons are $\delta E / E=$ $\sqrt{(0.135)^{2} / E_{T}+(0.02)^{2}}$ and $\delta P_{T} / P_{T}=0.0011 \times$ $P_{T}(\mathrm{GeV} / c)$, where $E_{T}$, in $\mathrm{GeV}$, is the electron energy measured in the calorimeter and $P_{T}$ is the beamconstrained muon transverse momentum measured in the CTC. For jets we use an $E_{T}$ dependent Gaussian resolution appropriate for $b$ partons derived from the HERWIG $t \bar{t}$ Monte Carlo, in conjunction with detector simulation, assuming a top mass of $170 \mathrm{GeV} / c^{2}$ [10]. The $\mathbb{E}_{T}$ is recomputed for each sampling using the new jet and lepton energies. This procedure takes into account all the uncertainties in the $\mathscr{E}_{T}$ measurement except for the resolution of the unclustered energy measurement, which is estimated to be $4 \mathrm{GeV}$ for both transverse components for low luminosity events. Hence we use $\sigma=4 \sqrt{n} \mathrm{GeV}$ in the above expression for $g\left(m_{t}, \eta_{1}, \eta_{2}\right)$, where $n$ is the number of interactions in the event. This value has been obtained from minimum bias events and for each event is properly scaled to take into account the effect of multiple interactions at high luminosity.

For each assumed top mass value we use 100 values of the two neutrinos $\eta_{1}$ and $\eta_{2}$, chosen from distributions obtained from the HERWIG Monte Carlo predictions [11]. They are consistent with independent Gaussian distributions with $\sigma=1.0$ unit of $\eta$. The weight is summed for all samplings, over all $\eta_{1}, \eta_{2}$ values, and all the eight possible combinations; thus for each event at each top mass, $m_{t}$, we evaluate an overall weight [5],

$$
f\left(m_{t}\right)=\sum_{\eta_{1}, \eta_{2}, E_{T_{i}}, \ell_{1}, \ell_{2}} g\left(m_{t}, \eta_{1}, \eta_{2}\right),
$$

where $E_{T_{i}}$ refers to all the jets in the event. We then compute the weight as a function of the top mass in $2.5 \mathrm{GeV} / c^{2}$ steps in the range $90-290 \mathrm{GeV} / c^{2}$.

The $f\left(m_{t}\right)$ distribution for each of the eight candidate events, normalized to unity, is shown in Fig. 1. For each event, $i$, we use this distribution to determine a top mass estimate, $m_{i}$, by averaging the values of $m_{t}$ corresponding to values of $f\left(m_{t}\right)$ closest to and greater than $f\left(m_{t}\right)_{\max } / 2$ on either side of the maximum. The $m_{i}$ distribution for the eight events is shown in Fig. 2, together with the Monte Carlo expectation for background alone, and top plus background normalized to the data.

Given the $m_{i}$ distribution for a sample of $N$ events, we use a maximum likelihood method similar to that employed in the lepton plus jets mass analysis [10] to extract a top mass value. We write the likelihood as

$$
\begin{aligned}
\mathcal{L}= & G\left(n_{b} ; \bar{n}_{b}, \sigma_{b}\right) \cdot P\left(N ; n_{s}+n_{b}\right) \\
& \times \prod_{i} \frac{n_{s} T\left(m_{i}, m_{t}\right)+n_{b} B\left(m_{i}\right)}{n_{s}+n_{b}},
\end{aligned}
$$

where $T\left(m_{i}, m_{t}\right)$ and $B\left(m_{i}\right)$ are the probability density functions (templates) for reconstructing a mass $m_{i}$ from $t \bar{t}$ events with a true top mass $m_{t}$ and from background events, respectively; $n_{s}$ and $n_{b}$ are the numbers of $t \bar{t}$

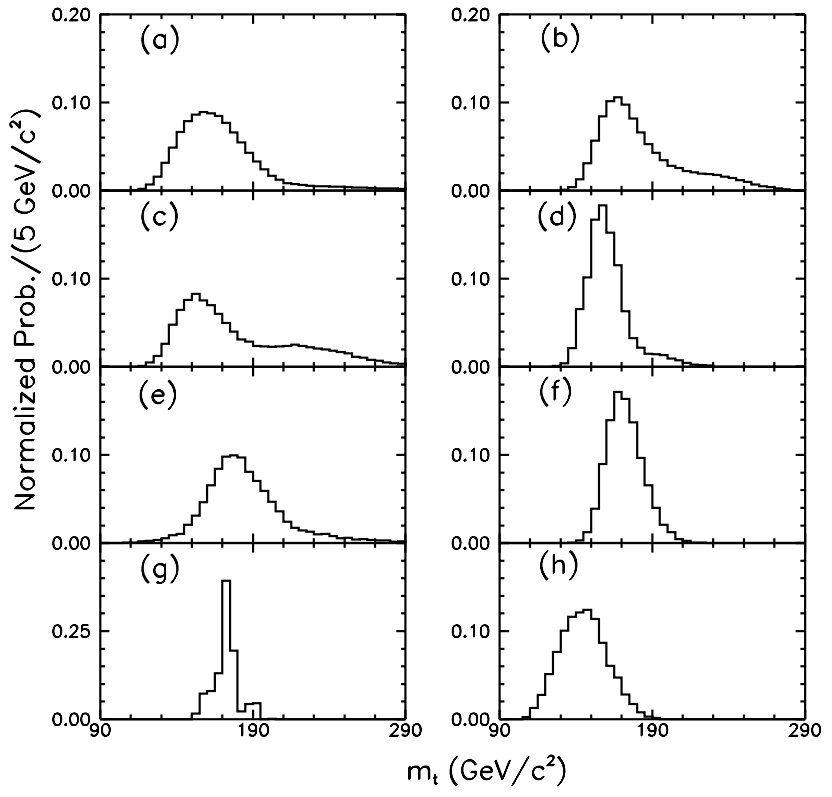

FIG. 1. Weight distribution normalized to unity as a function of $m_{t}$ for the eight dilepton top candidate events (a)-(h).

and background events; $G\left(n_{b} ; \bar{n}_{b}, \sigma_{b}\right)$ is a Gaussian in $n_{b}$ with mean $\bar{n}_{b}$ (1.3 events) and width $\sigma_{b}$ (0.3 events), and $P\left(N ; n_{s}+n_{b}\right)$ is a Poisson distribution in $N$ with mean $n_{s}+n_{b}$. The top templates, $T\left(m_{i}, m_{t}\right)$, are obtained by performing the dilepton mass reconstruction algorithm on HERWIG $t \bar{t}$ Monte Carlo samples, and parametrizing the resulting reconstructed mass distributions as smooth functions of both $m_{i}$ and $m_{t}$ [2]. The background templates, $B\left(m_{i}\right)$, are derived from data and Monte Carlo

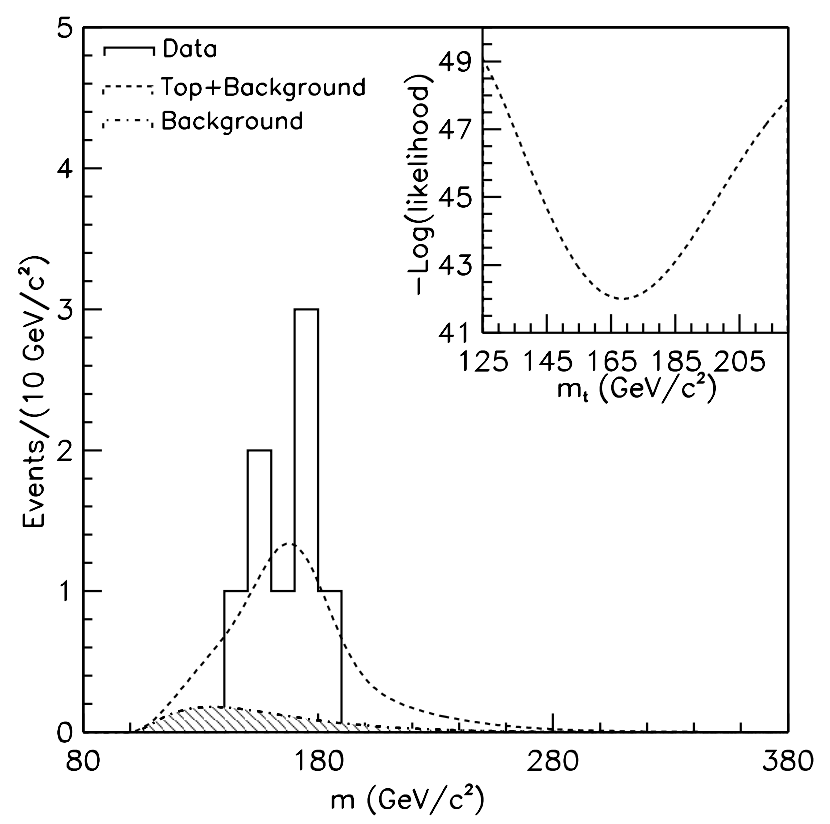

FIG. 2. Reconstructed top mass for the eight dilepton events (solid line). Background distribution (shaded region, 1.3 events) and top Monte Carlo (6.7 events) added to background (dashed curve). The negative log-likelihood distribution as a function of the top mass is shown in the inset. 
[1]. The likelihood fit gives the top mass measurement and a statistical error taken from the change in the $-\log (\mathcal{L})$ of 0.5 units from its minimum value.

To check the likelihood fitting technique, we use Monte Carlo events to perform many pseudoexperiments for several input top mass values. At each top mass value each experiment consists of a total of eight top and background events, with the number of background events drawn from a binomial distribution with a mean of $1.3 \pm$ 0.3 events. The mass estimate for each event is drawn from the appropriate template. For each experiment we obtain a mass, $m_{\exp }$, and a statistical error, $\sigma_{\exp }$. We study the distributions of the pulls, $\left(m_{\exp }-m_{t}\right) / \sigma_{\text {exp }}$, and find that the medians are consistent with zero and the widths are at most 1.1. We take this width into account by multiplying the error returned by the fit by a factor of 1.1. This factor is included in all statistical errors on the dilepton measurements given below.

We apply the likelihood method to the data shown in Fig. 2. The inset shows the negative log-likelihood as a function of the top mass, from which we determine a top quark mass value of $167.4 \pm 10.3$ (stat) $\mathrm{GeV} / \mathrm{c}^{2}$. Monte Carlo studies performed with pseudoexperiments yield an $8 \%$ probability for one such experiment to have a statistical error $\leq 10.3 \mathrm{GeV} / c^{2}$ at a mass of $167 \mathrm{GeV} / c^{2}$. In comparison, the previous result was $161 \pm 17$ (stat) $\mathrm{GeV} / c^{2}[1]$.

The mass reconstruction procedure used here is quite different from that used for the lepton plus jets sample [2]. In that case there is only one missing neutrino, and a kinematic fit with two constraints can be performed. We cross-check the present procedure by applying it to the five events of the lepton plus four jets mass sample in which two jets are tagged as $b$ jets by the SVX [2]. We assume that the two untagged jets are the products of the hadronic $W$ decay and mimic the dilepton decay by treating the highest $E_{T}$ untagged jet as a lepton and the second untagged jet as a neutrino. The present likelihood method then gives a top quark mass from the five events of $181.5 \pm 12.6 \mathrm{GeV} / c^{2}$, which differs by $11 \mathrm{GeV} / c^{2}$ from that obtained with the lepton plus jets kinematic fit procedures, $170.1 \pm 9.3 \mathrm{GeV} / c^{2}[2]$. In comparison, Monte Carlo studies show that the difference in mass obtained with the two methods is expected to be centered at zero with a resolution of $14 \mathrm{GeV} / c^{2}$.

The systematic errors on the mass measurement are estimated with the same general procedure as in the lepton plus jets mass analysis [2]: we generate a new top mass distributions by varying the appropriate quantities in the Monte Carlo simulation and then perform likelihood fits to many experiments of eight events each using the standard templates. The mass shifts obtained determine the systematic errors, which are summarized in Table I. The largest contribution comes from the systematic uncertainties on the jet energy. The uncertainty in modeling initial or final state radiation in $t \bar{t}$ events is estimated using events generated with the PYTHIA Monte
TABLE I. Systematic errors (in $\mathrm{GeV} / c^{2}$ ) on the top mass measurement for the three $t \bar{t}$ decay channels. The Monte Carlo modeling term includes effects from parton distribution functions and $b$-tag uncertainty where applicable. For the lepton plus jet channel the Monte Carlo statistics term was included in the statistical error.

\begin{tabular}{lccc}
\hline \hline \multicolumn{1}{c}{ Channel } & Dilepton & $\ell+$ jets & All-hadronic \\
\hline Jet energy scale & 3.8 & 4.4 & 5.0 \\
Initial and final & & & \\
$\quad$ state radiation & 2.7 & 2.6 & 1.8 \\
Monte Carlo modeling & 0.6 & 0.5 & 0.2 \\
Monte Carlo generator & 0.6 & 0.1 & 0.8 \\
Background shape & 0.3 & 1.3 & 1.7 \\
Monte Carlo statistics & 0.7 & $\ldots$. & 0.6 \\
Total & 4.8 & 5.3 & 5.7 \\
\hline \hline
\end{tabular}

Carlo program [12] to isolate the effects on the top mass due to initial and final state radiation jets. The error due to background shape is estimated by refitting the data with three different backgrounds: $W^{+} W^{-}$, fake leptons, and their weighted sum. That due to the choice of parton distribution functions $\left(0.6 \mathrm{GeV} / c^{2}\right)$ is estimated by using an alternative set of functions in the HERWIG Monte Carlo. The effect due to the choice of Monte Carlo generator $\left(0.6 \mathrm{GeV} / c^{2}\right)$ is estimated by comparing the mass value obtained with events from the HERWIG and PYTHIA Monte Carlo samples. The total systematic error amounts to $4.8 \mathrm{GeV} / c^{2}$.

Table I includes the systematic errors for the other two decay channels. The systematic error due to hard gluon radiation uncertainty in the lepton plus jets [2] and the all-hadronic [3] channels are now evaluated in the same way as described above. For the all-hadronic case the old value of $8.0 \mathrm{GeV} / c^{2}$ was an overly conservative estimate. Also, the "fit procedure" systematic in the all-hadronic channel $\left(5.0 \mathrm{GeV} / c^{2}\right)$ has been removed as being overly conservative. The overall systematic error for the lepton plus jets channel has increased from 4.9 to $5.3 \mathrm{GeV} / c^{2}$, and that for the all-hadronic channel is reduced from 12.0 to $5.7 \mathrm{GeV} / c^{2}$. The new top mass measurement for the dilepton channel and those for the other two channels with revised systematic errors are shown in Table II.

The results for the three channels are combined with standard methods [13] to yield an overall CDF mass measurement. The three statistical errors are taken as uncorrelated, while the systematic errors are assumed to be either entirely correlated or uncorrelated between any two channels. The primary systematic error, that due to jet energy uncertainty, is taken as entirely correlated among all channels, as is the systematic error due to the Monte Carlo model used (gluon radiation and Monte Carlo entries in Table I). The combined result is

$$
m_{t}=176.0 \pm 6.5 \mathrm{GeV} / c^{2},
$$

including both statistical and systematic errors; see Table II. The relative contributions from the three channels are $67 \%$ for lepton plus jets, $18 \%$ for dileptons, and $15 \%$ 
TABLE II. Summary of top mass measurements with the CDF detector. For the combined mass the statistical error is defined as the sum in quadrature of the weighted individual statistical errors, and the systematic error as the difference in quadrature of the total and statistical errors.

\begin{tabular}{llc}
\hline \hline \multicolumn{1}{c}{ Channel } & Top mass $\left(\mathrm{GeV} / c^{2}\right)$ & Reference \\
\hline Dilepton & $167.4 \pm 10.3 \pm 4.8$ & this paper \\
Lepton + jets & $175.9 \pm \sim 4.8 \pm 5.3$ & [2], this paper \\
All-hadronic & $186.0 \pm 10.0 \pm 5.7$ & [3], this paper \\
Combined & $176.0 \pm \sim 4.0 \pm 5.1$ & this paper \\
\hline \hline
\end{tabular}

for all-hadronic. The above top quark mass value agrees with the D0 measurement of $172.1 \pm 7.1 \mathrm{GeV} / c^{2}[14]$.

We thank the Fermilab staff and the technical staffs of the participating institutions for their contributions. This work was supported by the U.S. Department of Energy and National Science Foundation, the Italian Istituto Nazionale di Fisica Nucleare, the Ministry of Science, Culture, and Education of Japan, the Natural Sciences and Engineering Research Council of Canada, the National Science Council of the Republic of China, and the A. P. Sloan Foundation.

*Visitor.

[1] F. Abe et al., Phys. Rev. Lett. 80, 2779 (1998).

[2] F. Abe et al., Phys. Rev. Lett. 80, 2767 (1998).

[3] F. Abe et al., Phys. Rev. Lett. 79, 1992 (1997).
[4] B. Abbott et al., Phys. Rev. Lett. 79, 1197 (1997).

[5] B. Abbott et al., Phys. Rev. Lett. 80, 2063 (1998).

[6] F. Abe et al., Nucl. Instrum. Methods Phys. Res., Sect. A 271, 387 (1988).

[7] D. Amidei et al., Nucl. Instrum. Methods Phys. Res., Sect. A 350, 73 (1994); P. Azzi et al., Nucl. Instrum. Methods Phys. Res., Sect. A 360, 137 (1995).

[8] In the CDF coordinate system, $\theta$ and $\phi$ are the polar and azimuthal angles, respectively, with respect to the proton beam direction which defines the $z$ axis. The pseudorapidity $\eta$ is defined as $-\ln \left(\tan \frac{\theta}{2}\right)$. The transverse momentum of a particle with momentum $P$ is $P_{T}=$ $P \sin \theta$. The analogous quantity using energy, defined as $E_{T}=E \sin \theta$, is called transverse energy. The missing transverse energy $\mathscr{E}_{T}$ is defined as $-\Sigma E_{T}^{i} \hat{r}_{i}$, where $\hat{r}_{i}$ is a unit vector in the transverse plane pointing to the center of calorimeter tower $i$ and $E_{T}^{i}$ is the transverse energy deposited in that tower. Only towers with $|\eta|<3.6$ are included.

[9] K. Kondo, J. Phys. Soc. Jpn. 57, 4126 (1988); 60, 836 (1991); R. H. Dalitz and G. R. Goldstein, Phys. Rev. D 45, 1531 (1992).

[10] F. Abe et al., Phys. Rev. D 50, 2966 (1994).

[11] G. Marchesini and B. Webber, Nucl. Phys. B310, 461 (1988). HERWIG version 5.6 was used in this analysis.

[12] T. Sjöstrand, Comput. Phys. Commun. 82, 74 (1994). PYTHIA version 5.7 was used in this analysis.

[13] Particle Data Group, R. M. Barnett et al., Phys. Rev. D 54, 1 (1996), formalism discussed on p. 161.

[14] B. Abbott et al., Fermilab Report No. FERMILAB-Pub98/261-E (to be published). 